\title{
INTERFERÊNCIA DE Raphanus sativus SOBRE Cultivares DE SOJA Durante a Fase Vegetativa de Desenvolvimento da Cultura ${ }^{1}$
}

\author{
Raphanus sativus Interference on Soybean Cultivars During Crop Vegetative Period
}

FLECK, N.G. ${ }^{2}$, BIANCHI, M.A. ${ }^{3}$, RIZZARDI, M.A. ${ }^{4}$ e AGOSTINETTO, D. ${ }^{5}$

\begin{abstract}
RESUMO - A caracterização da habilidade competitiva da soja, em função da resposta diferencial dos cultivares, pode fornecer subsídios valiosos tanto para o melhoramento genético como para o manejo de plantas daninhas. Várias características da cultura podem associar-se com a competitividade; contudo, em soja, poucos estudos foram realizados com a finalidade de identificá-las. Com os objetivos de avaliar a variabilidade existente em cultivares de soja quanto à competitividade com plantas concorrentes e identificar aqueles portadores de habilidade competitiva superior, foi conduzido experimento a campo, em Cruz Alta-RS, na safra 2000/ 01. Testaram-se duas condições de competição (ausência ou presença de nabo-forrageiro durante a fase de desenvolvimento vegetativo da soja), combinadas com 11 cultivares da cultura. A presença do nabo durante os primeiros 60 dias do ciclo da soja reduziu estatura de planta, área foliar, massa da parte aérea seca, emissão e crescimento de ramos. Os cultivares de soja responderam diferentemente à interferência do nabo, indicando existir variabilidade genética para competitividade, o que permite selecionar genótipos de soja portadores de habilidade competitiva superior. De outra parte, ocorreu maior supressão do crescimento de plantas de nabo em função da presença dos cultivares de soja 'CD 201' e 'Fepagro RS 10'.
\end{abstract}

Palavras-chave: Glycine max, habilidade competitiva, genótipos, nabo-forrageiro.

\begin{abstract}
The characterization of soybean competitive ability, as a function of cultivar differential response, may provide valuable information for genetic crop improvement as well as weed management. Various crop characteristics may be associated with competitivity; however, in soybean, only a few studies have been carried out to identify them. A field experiment was conducted in Cruz Alta-RS during 2000/2001 to evaluate variability in soybean cultivars for competitivity against concurrent plants and to identify those holding superior competitive ability. Two competition conditions were tested (absence and presence of forage radish during the period of soybean vegetative development), combined with 11 soybean cultivars. The presence of forage radish during the first 60 days of crop development reduced soybean plant height, leaf area, shoot dry weight, and branch emission and growth. Soybean cultivars reacted differently to forage radish interference, suggesting the existence of genetic variability for competitivity, which allows for selection of soybean genotypes holding superior competitive ability. On the other hand, a greater growth suppression of forage radish plants was verified when associated with soybean cultivars 'CD 201' and 'Fepagro RS 10'.
\end{abstract}

Keywords: Glycine max, competitive ability, genotypes, forage radish.

1 Recebido para publicação em 30.11.2005 e na forma revisada em 4.8.2006.

Parte da tese do segundo autor para obtenção do título de Doutor em Fitotecnia, pelo PPG Fitotecnia, Faculdade de Agronomia, Universidade Federal do Rio Grande do Sul - UFRGS.

2 Eng.-Agr., Prof. do Departamento de Plantas de Lavoura, Faculdade de Agronomia - UFRGS, Bolsista de Pesquisa do CNPq, <fleck@ufrgs.br>; ${ }^{3}$ Aluno do PPG Fitotecnia - UFRGS, Eng.-Agr., Pesquisador da Fundacep, Caixa Postal 10, $98100-970$ Cruz Alta-RS, <mariobianchi@fundacep.com.br>; ${ }^{4}$ Eng.-Agr., Prof. da Faculdade de Agronomia e Medicina Veterinária - UPF, <rizzardi@upf.tche.br>; ${ }^{5}$ Eng.-Agr., Prof. do Departamento de Fitossanidade-FAEM/UFPel, <dirceu_agostinetto@ufpel.tche.br>.

Planta Daninha, Viçosa-MG, v. 24, n. 3, p. 425-434, 2006 


\section{INTRODUÇÃO}

O manejo de plantas daninhas com base no uso de herbicidas tem proporcionado o desenvolvimento de sistemas de produção relativamente simples, permitindo o aumento de áreas cultivadas. Por outro lado, a forte dependência das culturas no emprego de herbicidas gera ambientes favoráveis ao surgimento de plantas daninhas resistentes, mudança da flora daninha e movimento de herbicidas para locais não-alvos (Buhler, 2002). O manejo integrado de plantas daninhas antecipa-se a esses problemas, retirando os herbicidas do eixo central do manejo e preservando sua eficiência no controle de espécies infestantes.

Estudos sobre competitividade de culturas com plantas daninhas permitem desenvolver estratégias para sua inserção em sistemas de manejo integrado. Nos últimos anos, foram intensificadas as pesquisas direcionadas a definir características que confiram maior habilidade competitiva (HC), principalmente nas culturas de arroz e trigo e, em menor proporção, de milho e soja. A HC se caracteriza pela dominância de um indivíduo sobre seus vizinhos, os quais utilizam, simultaneamente, um mesmo recurso com limitada disponibilidade (Aarssen, 1983). A HC pode ser analisada, quanto aos efeitos, sob dois aspectos: supressão do crescimento de vizinhos e tolerância à presença de vizinhos (Goldberg \& Landa, 1991). Em sistemas agrícolas, o efeito supressivo deve preponderar em relação à tolerância das culturas, por reduzir a matéria seca e a produção de sementes das plantas daninhas e beneficiar seu manejo nas culturas subseqüentes (Jordan, 1993).

Geralmente, maior tamanho de planta exprime competitividade superior, sendo a matéria seca freqüentemente utilizada como indicadora de maior HC. Plantas portadoras de estatura elevada (Fleck, 1980; Shilling et al.,1995; McDonald, 2003), folhas amplas (Wortmann, 1993; Shilling et al., 1995; Lemerle et al., 1996), alto índice de área foliar - IAF (Wortmann, 1993; Gibson et al., 2003; Haefele et al., 2004) e elevada interceptação da radiação fotossinteticamente ativa (Lindquist \& Mortensen, 1998) identificam culturas com elevado potencial competitivo com plantas daninhas. Por sua vez, crescimento inicial vigoroso, expresso em matéria seca de plantas (Wortmann, 1993; Balbinot Jr. et al., 2003) e rápido incremento em estatura (Jannink et al., 2000; Caton et al., 2003), confere maior potencial competitivo às culturas. Em soja, a estatura de planta na fase inicial de crescimento apresenta alta herdabilidade e correlação genética com supressão de plantas daninhas, constituindo-se em critério ideal para seleção de plantas mais competitivas (Jannink et al., 2001).

A cobertura vegetal precoce do solo pelas espécies cultivadas suprime o crescimento de plantas daninhas (Wax \& Pendleton, 1968). A cobertura do solo por plantas de arroz foi a característica que se relacionou bem com competitividade, estando esta associada positivamente com a velocidade de emergência, área foliar, estatura e matéria seca das plantas de arroz (Balbinot Jr. et al., 2003).

A arquitetura da planta exerce influência sobre sua capacidade de competição. Maior concentração de folhas acima da espiga resultou em menor prejuízo ao milho devido à competição com espécies daninhas (Begna et al., 2001). A concentração de folhas na parte superior da planta indica distinta alocação de matéria seca entre os órgãos, mecanismo que pode estar envolvido com tolerância às ervas. Nesse sentido, cultivares mais tolerantes à interferência por plantas daninhas apresentam maior partição de matéria seca para folhas nos estádios iniciais de crescimento (Lindquist \& Mortensen, 1999) e maior densidade de folhas na parte superior da planta (Traoré et al., 2002).

O crescimento inicial mais vigoroso de cultivares precoces pode ser decisivo na resposta de sua competitividade com a flora daninha predominante. Isso assume grande importância em lavouras de soja no Sul do Brasil, pelo fato de a maturação das principais espécies daninhas ocorrer antes da colheita dos cultivares precoces de soja (Voll et al., 2002). Por outro lado, é viável selecionar cultivares tardios que apresentem rápido crescimento na fase inicial de desenvolvimento, sem que haja prejuízo em seu crescimento nos estádios mais avançados, conferindo-lhes, assim, alta competitividade durante todo o ciclo (Jannink et al., 2001). 
Os objetivos deste experimento foram avaliar a variabilidade em características de plantas de cultivares de soja para competitividade com plantas concorrentes e identificar aqueles portadores de habilidade competitiva superior.

\section{MATERIAL E MÉTODOS}

Conduziu-se um experimento a campo durante o ano agrícola 2000/01, em Cruz Alta (RS), na Fundação Centro de Experimentação e Pesquisa (FUNDACEP). O solo da área experimental apresenta as seguintes características físico-químicas: argila = 63\%; matéria orgânica $=3 \% ; \mathrm{pH}$ em água $=5,4 ; \mathrm{P}=19 \mathrm{mg} \mathrm{L}^{-1} ; \mathrm{e} \mathrm{K}=$ $140 \mathrm{mg} \mathrm{L}^{-1}$. No estudo, testaram-se duas condições de competição (ausência e presença do competidor), combinadas com 11 cultivares de soja [BR 16, Bragg, BRS 154, CD 201, CEPS 9678, CEPS 9703, Fepagro RS 10 (RS 10), FT 2000, Fundacep 38, IAS 5 e MSoy 6101]. Os tratamentos resultantes dessas combinações foram dispostos em delineamento em parcelas subdivididas, com as parcelas principais distribuídas em quatro blocos. Adicionalmente, em cada bloco, alocou-se uma unidade experimental contendo apenas a espécie competidora.

Foi escolhido como competidor Raphanus sativus (nabo ou nabo-forrageiro), por representar espécie que pode infestar a cultura de soja e por mostrar germinação e emergência mais uniformes do que espécies daninhas típicas. Essa característica garante densidade de plantas semelhante entre as unidades experimentais; em decorrência, a cultura é submetida à pressão de competição uniforme, proporcionando maior confiabilidade aos resultados (Fleck et al., 2003; McDonald, 2003).

A área experimental, manejada no sistema plantio direto, foi semeada no dia 17 de novembro de 2000, sendo as sementes de nabo (55 sementes aptas $\mathrm{m}^{-2}$ ) distribuídas em linhas espaçadas de 0,17 m, e as de soja (33 sementes aptas $\mathrm{m}^{-2}$ ), em linhas espaçadas de $0,5 \mathrm{~m}$. A semeadura do nabo precedeu a da soja, o qual foi semeado em sentido perpendicular ao das linhas da cultura. As práticas culturais adotadas seguiram as recomendações preconizadas para a cultura. O controle de plantas daninhas gramíneas foi efetuado com o herbicida clethodim (96 $\mathrm{g} \mathrm{ha}^{-1}$ ) e o das dicotiledôneas, exceto nabo, foi realizado por meio de arrancamento manual.

Na soja, foram efetuadas avaliações aos 15, 30, 45 e 60 dias após a emergência (DAE) das plantas, determinando-se: estatura de planta, massa da matéria seca da parte aérea (MPA) e das folhas, área foliar (AF), número de ramos (NR), comprimento médio de ramos (CMR) e cobertura vegetal do solo pelo dossel das plantas de soja (CS). Foram calculadas a razão de massa foliar (RMF) e a taxa de crescimento relativo (TCR) por meio das seguintes equações (Radosevich et al., 1997): RMF = MF/MPA; e $\mathrm{TCR}=\left(1 / \mathrm{MPA}_{\mathrm{i}}\right) *(\mathrm{dM} / \mathrm{dt})$, em que $M P A=$ massa da matéria seca da parte aérea; $M P A_{i}=$ massa da matéria seca inicial da parte aérea; $M F=$ massa da matéria seca foliar; $d M=$ incremento de matéria seca no intervalo de tempo considerado; e $d t$ = intervalo de tempo adotado. No nabo, foram avaliadas a população de plantas, MPA e produtividade de grãos. A colheita das plantas de nabo para produtividade de grãos foi realizada manualmente aos $60 \mathrm{DAE}$ da soja, na área útil das unidades experimentais, coincidindo com o florescimento da maioria dos cultivares de soja.

Quando o teste $\mathrm{F}$ da análise de variância indicou significância (efeito principal p d 0,05; interação p d 0,15), as médias foram comparadas pelo teste da DMS $(\mathrm{p}=0,05)$. A variável cobertura do solo pela soja e as variáveis determinadas em nabo, por representarem apenas uma das condições de competição, foram analisadas considerando-se o delineamento experimental em blocos casualizados. A comparação entre tratamentos das variáveis determinadas no nabo em monocultivo (testemunha) e na presença dos cultivares de soja foi efetuada pelo teste de Dunnett $(p=0,05)$. Para análise estatística utilizou-se o programa SAS (SAS Institute, 1989).

\section{RESULTADOS E DISCUSSÃO}

A presença de nabo reduziu, em média, 14 e $19 \%$ a estatura de planta dos cultivares de soja aos 45 e 60 DAE, respectivamente (Tabela 1). Os cultivares Bragg e RS 10 aos 15 DAE, Bragg e MSoy 6101 aos 30 DAE e MSoy 6101 aos 45 DAE destacaram-se como os mais altos. Por outro lado, os cultivares 
CD 201, FT 2000 e IAS 5 geralmente figuraram entre os mais baixos. Aos $60 \mathrm{DAE}$, independentemente da presença de nabo, MSoy 6101 apresentou a maior estatura, enquanto IAS 5 e FT 2000 repetiram suas posições de porte mais baixo.

As diferenças em estatura de planta aos 15 DAE, entre os cultivares RS 10 e Bragg, em comparação com CD 201, FT 2000 e IAS 5, podem ser atribuídas, em parte, à matéria seca média das sementes, que nessa época ainda pode ter influenciado o crescimento das plantas. Nesse caso, plantas altas podem associarse com matéria seca de sementes média a alta, e as mais baixas, com sementes de matéria seca baixa. Nas épocas posteriores de avaliação, a maior e a menor estatura de planta corresponderam, respectivamente, aos cultivares MSoy 6101 e IAS 5. Nota-se, também, que aos 60 DAE a diferença entre esses cultivares, na ausência e presença de nabo, indica que a competição não alterou seu padrão de crescimento em estatura. Esses cultivares se caracterizam por possuírem ciclo de crescimento precoce, mas diferem em hábito de crescimento (MSoy 6101 é indeterminado e IAS 5 é determinado) e estatura final (MSoy 6101 é alta e IAS 5 é média). Possivelmente, essas características justifiquem as diferenças em estatura verificadas entre eles em fases iniciais do ciclo.

Plantas altas, em geral, demonstram maior competitividade com infestantes, devido à vantagem obtida na captação de radiação solar em relação a espécies daninhas mais baixas, conforme verificado em trigo (Fleck, 1980), sorgo (Traoré et al., 2002) e ervilha (McDonald, 2003). Além disso, o rápido crescimento em estatura na fase inicial de desenvolvimento, como ocorreu neste trabalho com MSoy 6101, conferiu à soja maior competitividade com a espécie Brassica hirta (mostarda-branca) (Jannink et al., 2000).

A competição do nabo reduziu a $\mathrm{AF}$ das plantas de soja aos 30, 45 e $60 \mathrm{DAE}$ para a maioria dos cultivares, especialmente nas duas últimas avaliações (Tabela 2). Nas duas primeiras, o cultivar RS 10 não foi afetado pela competição do nabo, mantendo inalterada sua AF. Ao contrário, CD 201 foi o cultivar com a AF mais reduzida pela competição do nabo, consideradas as três avaliações. Características como tamanho de folhas, área foliar e

Tabela 1 - Estatura de plantas (cm) de soja aos 15, 30, 45 e 60 dias após a emergência (DAE), na ausência e presença de nabo. Cruz Alta-RS, 2001

\begin{tabular}{|c|c|c|c|c|c|}
\hline \multirow{2}{*}{ Fator } & \multirow{2}{*}{$15 \mathrm{DAE}$} & \multirow{2}{*}{$30 \mathrm{DAE}$} & \multirow{2}{*}{$45 \mathrm{DAE}$} & \multicolumn{2}{|c|}{$60 \mathrm{DAE}$} \\
\hline & & & & Sem nabo & Com nabo \\
\hline IAS 5 & $7,3 \mathrm{e}$ & $16,5 \mathrm{~d}$ & $38,6 \mathrm{~g}$ & A $66,2 \mathrm{f}$ & B $53,8 \mathrm{e}$ \\
\hline BR 16 & $8,9 \mathrm{~b}$ & $19,2 \mathrm{~b}$ & $45,5 \mathrm{bc}$ & A 76,6 bc & B 63,0 bc \\
\hline CD 201 & $6,9 \mathrm{e}$ & $16,6 \mathrm{~d}$ & 41,4 ef & A 78,9 b & B 58,1 de \\
\hline MSoy 6101 & $8,3 \mathrm{~cd}$ & $21,0 \mathrm{a}$ & $51,3 \mathrm{a}$ & A 88,8 a & B 74,7 a \\
\hline Bragg & $9,5 \mathrm{a}$ & $20,4 \mathrm{a}$ & $46,6 \mathrm{~b}$ & A $71,5 \mathrm{de}$ & B 64,4 b \\
\hline BRS 154 & $8,6 \mathrm{bc}$ & $19,1 \mathrm{~b}$ & $43,9 \mathrm{~cd}$ & A $75,4 \mathrm{bcd}$ & B $60,7 \mathrm{bcd}$ \\
\hline Fundacep 38 & $8,2 \mathrm{~cd}$ & $17,3 \mathrm{~cd}$ & $40,6 \mathrm{fg}$ & A 73,0 cde & B 56,7 de \\
\hline Fepagro RS 10 & $9,3 \mathrm{a}$ & $19,2 \mathrm{~b}$ & $44,4 \mathrm{~cd}$ & A $74,2 \mathrm{~cd}$ & B 63,0 bc \\
\hline CEPS 9703 & $8,3 \mathrm{~cd}$ & $18,4 \mathrm{bc}$ & $43,9 \mathrm{~cd}$ & A 76,6 bc & B $60,1 \mathrm{~cd}$ \\
\hline FT 2000 & $7,0 \mathrm{e}$ & $16,6 \mathrm{~d}$ & $39,1 \mathrm{~g}$ & A 69,0 ef & B 55,0 e \\
\hline CEPS 9678 & $8,1 \mathrm{~d}$ & $18,2 \mathrm{bc}$ & $43,2 \mathrm{de}$ & A 72,4 cde & B $60,0 \mathrm{~cd}$ \\
\hline Erros-padrão & $\pm 0,22$ & $\pm 0,55$ & $\pm 1,04$ & \multicolumn{2}{|c|}{ $\pm 2,16$} \\
\hline Sem nabo & $8,4^{\mathrm{ns}}$ & $18,2^{\mathrm{ns}}$ & $46,6 \mathrm{a}$ & \multicolumn{2}{|c|}{-} \\
\hline Com nabo & 8,1 & 18,6 & $40,4 \mathrm{~b}$ & \multicolumn{2}{|c|}{-} \\
\hline Erros-padrão & $\pm 0,15$ & $\pm 0,35$ & $\pm 0,72$ & & \\
\hline
\end{tabular}

Médias antecedidas pela mesma letra maiúscula nas linhas, entre condições de competição, ou seguidas pela mesma letra minúscula nas colunas, entre cultivares ou condições de competição, não diferem pelo teste da DMS ( $\mathrm{p}=0,05$ para efeito principal e $\mathrm{p}=0,15$ para interação de fatores).

${ }^{\text {ns }}$ Teste F não-significativo ( $\left.\mathrm{p}=0,05\right)$. 
Tabela 2 - Área foliar $\left(\mathrm{cm}^{2}\right.$ por planta) de plantas de soja aos 30, 45 e 60 dias após a emergência (DAE), na ausência e presença de nabo. Cruz Alta-RS, 2001

\begin{tabular}{|c|c|c|c|c|c|c|}
\hline \multirow{2}{*}{ Cultivar } & \multicolumn{2}{|c|}{$30 \mathrm{DAE}$} & \multicolumn{2}{|c|}{$45 \mathrm{DAE}$} & \multicolumn{2}{|c|}{$60 \mathrm{DAE}$} \\
\hline & Sem nabo & Com nabo & Sem nabo & Com nabo & Sem nabo & Com nabo \\
\hline IAS 5 & A $365 \mathrm{~cd}$ & B 228 a & A 1338 bcd & B 760 a & A 2578 efg & B $1380 \mathrm{~cd}$ \\
\hline BR 16 & A $356 \mathrm{~cd}$ & A 298 a & A 1166 de & B 753 a & A $2135 \mathrm{~g}$ & A 1686 abc \\
\hline CD 201 & A $479 \mathrm{~b}$ & B 229 a & A $1283 \mathrm{~cd}$ & B 514 ab & A 3247 bc & B 1418 bcd \\
\hline MSoy 6101 & A $362 \mathrm{~cd}$ & A 266 a & A $1273 \mathrm{~cd}$ & B $691 \mathrm{ab}$ & A 3056 bcde & B $1895 \mathrm{abc}$ \\
\hline Bragg & A $349 \mathrm{~cd}$ & A 297 a & A 1117 de & B 726 ab & A 2668 defg & B 1476 bcd \\
\hline BRS 154 & A 439 bc & B 255 a & A $1620 \mathrm{ab}$ & B $453 \mathrm{~b}$ & A 2841 cdef & B 1528 bcd \\
\hline Fundacep 38 & A 424 bc & B $250 \mathrm{a}$ & A 1513 abc & B 760 a & A 4167 a & B $1375 \mathrm{~cd}$ \\
\hline Fepagro RS 10 & A $367 \mathrm{~cd}$ & A 267 a & A $926 \mathrm{e}^{-}$ & A $735 \mathrm{ab}$ & A 3180 bcd & B $1916 \mathrm{ab}$ \\
\hline CEPS 9703 & A 402 bcd & B 250 a & A $1353 \mathrm{bcd}$ & B $677 \mathrm{ab}$ & A 2667 defg & B $1435 \mathrm{bcd}$ \\
\hline FT 2000 & A $300 \mathrm{~d}$ & A 201 a & A $1086 \mathrm{de}$ & B $625 \mathrm{ab}$ & A 2448 fg & B 1099 d \\
\hline CEPS 9678 & A 634 a & B 286 a & A 1700 a & B 815 a & A 3534 b & B 2078 a \\
\hline Erros-padrão & \multicolumn{2}{|c|}{ $\pm 40,6$} & \multicolumn{2}{|c|}{ $\pm 103,7$} & \multicolumn{2}{|c|}{ $\pm 176,5$} \\
\hline
\end{tabular}

Médias antecedidas pela mesma letra maiúscula nas linhas, entre condições de competição, ou seguidas pela mesma letra minúscula nas colunas, entre cultivares, não diferem pelo teste da DMS ( $\mathrm{p}=0,05$ para efeito principal e $\mathrm{p}=0,15$ para interação de fatores).

índice de área foliar costumam associar-se positivamente aos cultivares mais competitivos (Wortmann, 1993; Gibson et al., 2003). Dentre os cultivares testados, RS 10 se caracterizou por manter AF elevada na condição de competição. O desenvolvimento precoce de alta $\mathrm{AF}$ (Begna et al., 2001) e sua manutenção, mesmo sob competição, apontam cultivar com maior potencial competitivo.

Os cultivares responderam de forma diferenciada em MPA à presença de nabo no primeiro mês após a emergência (Tabela 3). Aos 45 DAE, a competição do nabo ocasionou redução de 56\%, em média, na MPA dos cultivares de soja. Já aos 60 DAE, embora o efeito negativo da presença do nabo ainda continuasse elevado (36\%), verificou-se certa recuperação da soja, possivelmente decorrente da aproximação do final do ciclo do nabo. O cultivar RS 10 destacou-se por produzir matéria seca elevada, tanto na ausência como na presença de nabo. Consideradas conjuntamente as duas últimas avaliações, os cultivares RS 10 e CEPS 9678 assumiram superioridade para a variável MPA, enquanto os cultivares CD 201 e FT 2000 foram os inferiores.

A matéria seca que uma planta acumula é o resultado da quantidade de recursos do ambiente que ela consegue captar. Em geral, plantas que produzem mais matéria seca causam maior depleção de recursos do meio, podendo resultar na supressão do crescimento de plantas vizinhas. O maior acúmulo de matéria seca nos cultivares RS 10 e CEPS 9678 sugere vantagem competitiva desses genótipos em relação a espécies daninhas. Em plantas de feijão, trigo e arroz, o rápido crescimento na fase inicial de desenvolvimento propiciou maior competitividade dessas espécies em relação às infestantes (Wortmann, 1993; Lemerle et al., 1996; Balbinot Jr. et al., 2003).

A emissão de ramos, medida aos $45 \mathrm{DAE}$, foi atrasada por efeito da competição do nabo (Tabela 4). Já aos 60 DAE houve equivalência no NR para a maioria dos cultivares, em função das condições de competição, exceto para CD 201 e Bragg, que mantiveram reduções na variável por efeito da presença de nabo. As diferenças no NR entre os cultivares, na presença de nabo, foram relativamente pequenas aos 45 DAE e ausentes aos 60 DAE. Na ausência do competidor, os cultivares que emitiram mais ramos foram RS 10 e Bragg aos 45 DAE e Bragg aos 60 DAE.

Para CMR não ocorreu interação dos fatores condições de competição e cultivares de soja (Tabela 4). A competição reduziu em 80 e $44 \%$ o CMR de plantas de cultivares de soja 
aos 45 e 60 DAE, respectivamente. Levandose em conta os valores absolutos obtidos, os cultivares BR 16 e IAS 5, aos 45 DAE, e CEPS 9678 e IAS 5, aos 60 DAE, apresentaram os maiores CMR, enquanto os menores CMR foram observados em MSoy 6101 aos 45 DAE e em CD 201 aos 60 DAE.
Plantas de soja com maior NR também tenderam a possuir ramos mais compridos, demonstrado pela associação positiva do NR com CMR aos 45 DAE ( $r=0,30 ; p=0,004)$ e aos 60 DAE $(r=0,32 ; p=0,002)$, o que pode resultar em maior matéria seca de ramos e em maior CS. Em arroz, plantas com mais matéria

Tabela 3 - Matéria seca da parte aérea (g por planta) de plantas de soja aos 15, 30, 45 e 60 dias após a emergência (DAE), na ausência e presença de nabo. Cruz Alta-RS, 2001

\begin{tabular}{|c|c|c|c|c|c|c|}
\hline \multirow{2}{*}{ Fator } & \multicolumn{2}{|c|}{$15 \mathrm{DAE}$} & \multicolumn{2}{|c|}{$30 \mathrm{DAE}$} & \multirow{2}{*}{$45 \mathrm{DAE}$} & \multirow{2}{*}{$60 \mathrm{DAE}$} \\
\hline & Sem nabo & Com nabo & Sem nabo & Com nabo & & \\
\hline IAS 5 & A 0,42 de & A 0,40 bcd & A 1,64 bc & B $1,02 a b$ & 5,6 bcd & $13,5 \mathrm{~cd}$ \\
\hline BR 16 & A 0,45 bcde & B 0,38 bcd & A $1,93 \mathrm{ab}$ & B $1,03 \mathrm{ab}$ & $6,2 \mathrm{abc}$ & $12,8 \mathrm{~d}$ \\
\hline CD 201 & A 0,43 cde & B $0,35 \mathrm{~d}$ & A 1,48 c & $\mathrm{B} 0,76 \mathrm{~b}$ & $5,4 \mathrm{~cd}$ & $12,7 \mathrm{~d}$ \\
\hline MSoy 6101 & A 0,43 cde & A 0,40 bcd & A 1,55 c & B $0,88 \mathrm{ab}$ & 5,8 bcd & $16,8 \mathrm{ab}$ \\
\hline Bragg & A 0,46 abcd & B $0,37 \mathrm{~cd}$ & A 1,42 c & B $0,90 \mathrm{ab}$ & $6,0 \mathrm{bcd}$ & $17,7 \mathrm{a}$ \\
\hline BRS 154 & A 0,49 abc & B 0,42 bc & A 1,62 bc & B $0,81 \mathrm{~b}$ & $5,5 \mathrm{~cd}$ & $14,5 \mathrm{bcd}$ \\
\hline Fundacep 38 & A 0,46 abcd & B $0,35 \mathrm{~d}$ & A 1,60 bc & B 0,72 b & $5,1 \mathrm{~d}$ & $14,4 \mathrm{bcd}$ \\
\hline Fepagro RS 10 & A 0,51 a & A 0,52 a & A 1,61 bc & B 1,20 a & $7,4 \mathrm{a}$ & $15,1 \mathrm{abcd}$ \\
\hline CEPS 9703 & A 0,48 abcd & $\mathrm{A} 0,44 \mathrm{~b}$ & A 2,06 a & B $1,01 \mathrm{ab}$ & $6,1 \mathrm{bcd}$ & $14,3 \mathrm{bcd}$ \\
\hline FT 2000 & A 0,40 e & A $0,36 \mathrm{~cd}$ & A 1,16 c & A 0,92 ab & $5,3 \mathrm{~cd}$ & $13,7 \mathrm{~cd}$ \\
\hline CEPS 9678 & A $0,50 \mathrm{ab}$ & B $0,36 \mathrm{~cd}$ & A $1,91 \mathrm{ab}$ & B $0,97 \mathrm{ab}$ & $6,6 \mathrm{ab}$ & $16,1 \mathrm{abc}$ \\
\hline Erros-padrão & \multicolumn{2}{|c|}{ $\pm 0,028$} & \multicolumn{2}{|c|}{ $\pm 0,179$} & $\pm 0,54$ & $\pm 1,46$ \\
\hline Sem nabo & \multicolumn{2}{|c|}{-} & \multicolumn{2}{|c|}{-} & 8,2 a & 17,9 a \\
\hline Com nabo & \multicolumn{2}{|c|}{-} & \multicolumn{2}{|c|}{-} & $3,6 \mathrm{~b}$ & $11,5 \mathrm{~b}$ \\
\hline Erros-padrão & & & & & $\pm 0,23$ & $\pm 0,66$ \\
\hline
\end{tabular}

Médias antecedidas pela mesma letra maiúscula nas linhas, entre condições de competição, ou seguidas pela mesma letra minúscula nas colunas, entre cultivares ou condições de competição, não diferem pelo teste da DMS ( $\mathrm{p}=0,05$ para efeito principal e $\mathrm{p}=0,15$ para interação de fatores).

Tabela 4 - Número de ramos (NR) e comprimento médio de ramos (CMR) de plantas de soja aos 45 e 60 dias após a emergência (DAE), na ausência e presença de nabo. Cruz Alta-RS, 2001

\begin{tabular}{|c|c|c|c|c|c|c|}
\hline \multirow{2}{*}{ Fator } & \multicolumn{2}{|c|}{ NR 45 DAE (ramos por planta) } & \multicolumn{2}{|c|}{ NR 60 DAE (ramos por planta) } & CMR 45 DAE & CMR 60 DAE \\
\hline & Sem nabo & Com nabo & Sem nabo & Com nabo & \multicolumn{2}{|c|}{ (cm por ramo) } \\
\hline IAS 5 & A 2,2 bc & B $0,3 \mathrm{ab}$ & A 4,2 bcde & A 3,7 a & $9,8 \mathrm{ab}$ & 19,4 a \\
\hline BR 16 & A 1,7 cdef & B $0,1 \mathrm{ab}$ & A 4,2 bcde & A 3,4 a & 13,3 a & 15,9 abcd \\
\hline CD 201 & A 1,4 def & $\mathrm{B} 0,0 \mathrm{~b}$ & A 4,7 bc & B 3,4 a & $4,6 \mathrm{~cd}$ & $11,2 \mathrm{e}^{-1}$ \\
\hline MSoy 6101 & A $0,6 \mathrm{~g}$ & $\mathrm{~A} 0,0 \mathrm{~b}$ & A 3,9 cde & A 3,8 a & $2,7 \mathrm{~d}$ & 16,6 abcd \\
\hline Bragg & A $2,9 \mathrm{ab}$ & B $0,3 \mathrm{ab}$ & A 6,5 a & B 4,2 a & $6,6 \mathrm{bc}$ & 15,6 abcd \\
\hline BRS 154 & A 2,2 bcd & $\mathrm{B} 0,0 \mathrm{~b}$ & A 5,1 b & A 4,2 a & $6,6 \mathrm{bc}$ & 15,2 bcde \\
\hline Fundacep 38 & A 1,3 efg & A $0,6 \mathrm{ab}$ & A 4,2 bcde & A 3,8 a & $4,1 \mathrm{~cd}$ & 16,9 abc \\
\hline Fepagro RS 10 & A 3,1 a & B $0,4 \mathrm{ab}$ & A 4,1 bcde & A 3,5 a & $7,6 \mathrm{bc}$ & $12,8 \mathrm{de}$ \\
\hline CEPS 9703 & A 1,8 cde & B 0,9 a & A 3,3 de & $\mathrm{A} 4,1 \mathrm{a}$ & $6,6 \mathrm{bc}$ & 14,8 bcde \\
\hline FT 2000 & $\mathrm{~A} 0,9 \mathrm{fg}$ & $\mathrm{B} 0,0 \mathrm{~b}$ & A $3,2 \mathrm{e}$ & A $3,5 \mathrm{a}$ & $4,0 \mathrm{~cd}$ & 13,1 cde \\
\hline CEPS 9678 & A 1,7 cdef & B $0,2 \mathrm{ab}$ & A 4,2 bcd & A 3,7 a & $5,4 \mathrm{~cd}$ & $17,3 \mathrm{ab}$ \\
\hline Erros-padrão & \multicolumn{2}{|c|}{ $\pm 0,38$} & \multicolumn{2}{|c|}{ $\pm 0,51$} & $\pm 1,90$ & $\pm 2,07$ \\
\hline Sem nabo & \multicolumn{2}{|c|}{-} & \multicolumn{2}{|c|}{-} & $10,8 \mathrm{a}$ & $19,7 \mathrm{a}$ \\
\hline Com nabo & \multicolumn{2}{|c|}{ 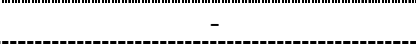 } & \multicolumn{2}{|c|}{-} & $2,2 \mathrm{~b}$ & $11,0 \mathrm{~b}$ \\
\hline Erros-padrão & \multicolumn{2}{|c|}{ 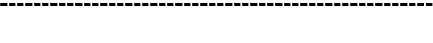 } & & & $\pm 0,81$ & $\pm 0,88$ \\
\hline
\end{tabular}

Médias antecedidas pela mesma letra maiúscula nas linhas, entre condições de competição, ou seguidas pela mesma letra minúscula nas colunas, entre cultivares ou condições de competição, não diferem pelo teste da DMS ( $\mathrm{p}=0,05$ para efeito principal e p=0,15 para interação de fatores). 
seca e maior CS pelo dossel possuem maior competitividade (Balbinot Jr. et al., 2003). No entanto, nesse estudo não houve associação da CS com NR ( $r=0,12 ; p=0,42)$ ou CMR ( $r=$ $0,05 ; p=0,74)$. Ressalte-se que o possível efeito benéfico das ramificações de plantas de soja sobre a HC foi minimizado pelo fato de a emissão e o crescimento dos ramos terem ocorrido tardiamente, em relação ao início da competição, e a competição com nabo ter sido efetiva somente durante a fase vegetativa da soja.

A RMF, em geral, aumentou quando os cultivares de soja cresceram na presença de nabo (dados não apresentados), indicando que maior quantidade de fotoassimilados foi direcionada às folhas para que as plantas superassem a competição. Em geral, destacaram-se com maiores valores absolutos de RMF os cultivares CEPS 9678, seguido por CD 201 e IAS 5, enquanto, negativamente, posicionaram-se os cultivares BR 16, RS 10, MSoy 6101 e FT 2000. Por outro lado, a TCR foi reduzida em $23,5 \%$ quando os cultivares de soja estiveram submetidos à competição com nabo (dados não apresentados). Devido à relação direta entre a matéria seca produzida por uma planta e o total de recursos utilizados durante o crescimento, a taxa de crescimento relativo pode ser usada como indicadora de HC potencial entre culturas e ervas (Radosevich et al.,1997).

Na avaliação da competitividade por meio da TCR deve ser levada em conta a matéria seca inicial e final da planta. Por exemplo, o cultivar RS 10 apresentou baixa TCR, mas possui os maiores valores de matéria seca inicial, e a matéria seca aos $60 \mathrm{DAE}$ é equivalente à dos cultivares com alta TCR (Tabela 3). Ao contrário, o cultivar FT 2000, mesmo com TCR equivalente à dos cultivares com os maiores valores, apresentou uma das menores MPA aos 15 e 60 DAE. Por sua vez, MSoy 6101, que inicialmente tinha uma das menores MPA, recuperou-se, chegando aos 60 DAE com um dos maiores valores dessa variável (Tabela 3). Isso demonstra que, mesmo com alta TCR, uma planta pode acumular menos matéria seca que outra com baixa TCR, devendo, portanto, ser tomadas em conjunto essas características, a fim de discriminar cultivares quanto à competitividade.

A cobertura do solo por plantas de soja é o resultado da expressão conjunta de várias características, como estatura, área foliar, matéria seca e ramificações (número, comprimento e distribuição) da planta. A cobertura do solo, medida na ausência de nabo, incrementou rapidamente no primeiro mês após a emergência da soja. Em média, a CS pelo dossel da soja foi de $18 \%$ aos $15 \mathrm{DAE}, 63 \%$ aos $30 \mathrm{DAE}, 87 \%$ aos $45 \mathrm{DAE}$ e $93 \%$ aos $60 \mathrm{DAE}$. A CS aos 45 DAE associou-se positivamente com MPA da soja aos 30 DAE ( $r=0,33 ; p=$ 0,03 ), indicando que plantas portadoras de mais matéria seca propiciam maior cobertura do solo. Culturas mais competitivas se caracterizam por rápida cobertura de solo (Wax \& Pendleton, 1968). No entanto, as amplitudes nos valores de CS ocorridas entre os cultivares foram relativamente pequenas $(8,13,8$ e $4 \%$, respectivamente, aos 15, 30, 45 e $60 \mathrm{DAE}$ ), reduzindo a probabilidade de essa variável contribuir decisivamente para diferenciá-los quanto à HC. Em situações mais contrastantes de CS por plantas de soja, a contribuição dessa variável na discriminação de cultivares poderá ter maior peso na HC.

A variação em estádio fenológico dos cultivares (dados não apresentados) não resultou em diferenças consistentes nas características de plantas avaliadas. Por exemplo, RS 10 e BRS 154, cultivares extremos quanto ao estádio fenológico, em geral, se equivaleram em estatura de planta e MPA (exceto aos 45 DAE) (Tabelas 1 e 3). Desse modo, grande parte das diferenças ocorridas em características morfológicas são atribuiveis às variações genéticas intrínsecas a cada cultivar, antes que às diferenças em estádios de desenvolvimento.

A população de plantas de nabo foi uniforme nas unidades experimentais, condição importante para garantir uma avaliação consistente de variabilidade na competitividade dos cultivares de soja (Tabela 5). A MPA do nabo aos 30 DAE não se alterou em função da presença da soja, mas a presença de soja reduziu a MPA do nabo aos 60 DAE. Nesse aspecto, os cultivares que causaram maior supressão no crescimento do competidor foram CD 201 (42\%), IAS 5 (40\%) e RS 10 (39\%); já a menor supressão ocorreu em presença de BRS 154 (22\%), Fundacep 38 (25\%) e CEPS 9703 (26\%). Todos os cultivares de soja reduziram a produtividade de grãos de nabo de 35 a $66 \%$, exceto BRS 154. As maiores quedas na 
produtividade de grãos de nabo associaram-se aos cultivares RS 10 (66\%) e IAS 5 (59\%). Combinando a MPA aos 60 DAE e a produtividade de grãos de nabo, constata-se que os cultivares RS 10 e IAS 5 causaram as maiores reduções nessas variáveis e BRS 154, seguido por Fundacep 38, as menores, nessas variáveis.

É de se supor que a maior parte do prejuízo causado pelo nabo não se deveu à disputa pela radiação solar fotossinteticamente ativa incidente no dossel da soja. No primeiro mês de crescimento da soja, a estatura de planta não foi afetada pela presença de nabo (Tabela 1), indicando que recursos do solo estiveram envolvidos na competição inicial. Em geral, plantas que competem por luz tendem a crescer mais em estatura para obter vantagem na captação desse recurso do ambiente (Rajcan \& Swanton, 2001). O nabo caracterizou-se por curto ciclo de crescimento (60 dias), o que implica que ele supriu toda sua demanda por recursos do solo durante esse período, restando menores quantidades para atender às demandas da soja. A cultura manifestou esse efeito reduzindo a MPA, a qual foi mais afetada aos 45 DAE. Crescimento inicial vigoroso, em estatura ou em matéria seca, indica plantas mais competitivas com plantas daninhas (Jannink et al., 2001).
As características de planta relacionadas com HC superior, referidas por vários autores em diferentes culturas, e a aceitação do cultivar pelo produtor permitem especular que o genótipo de soja detentor de potencial competitivo com plantas daninhas seria aquele que, mesmo sob estresse competitivo, mantivesse elevada e rápida a acumulação de matéria seca na fase inicial de crescimento, alta capacidade de ramificação, ramificação precoce, alocação preferencial de matéria seca para os ramos, estatura elevada, tolerância ao acamamento, baixa redução de produtividade sob estresse de competição e potencial de rendimento comparável ao dos cultivares existentes no mercado.

Levando em conta a habilidade competitiva e o potencial de o agricultor utilizar um cultivar mais produtivo, elaborou-se a seguinte classificação, em ordem preferencial: CD 201 > MSoy 6101 > RS 10 > IAS 5. Dificilmente uma única característica conferirá elevada habilidade competitiva às plantas de um cultivar (Lemerle et al., 1996), mas sim a combinação de duas ou mais delas, que, necessariamente, não são as mesmas entre cultivares. Considerando que a supressão do crescimento dos concorrentes, um dos enfoques dados à HC (Goldberg \& Landa, 1991), deve preponderar sobre a própria tolerância à competição

Tabela 5 - População de plantas (plantas $\mathrm{m}^{-2}$ ), matéria seca da parte aérea (MPA - $\mathrm{g} \mathrm{m}^{-2}$ ) aos 30 e 60 dias após a emergência (DAE) e produtividade de grãos de nabo $\left(\mathrm{kg} \mathrm{ha}^{-1}\right)$, crescendo em associação ou não com cultivares de soja. Cruz Alta-RS, 2001

\begin{tabular}{|c|c|c|c|c|c|c|}
\hline Cultivar & População & MPA 30 DAE & MPA 60 DAE & $\mathrm{R}_{\mathrm{MPA}^{1}}{ }^{\frac{1 /}{(}}(\%)$ & Produtividade & $\mathrm{R}_{\mathrm{PROD}^{1 /}}(\%)$ \\
\hline IAS 5 & $44^{\mathrm{ns}}$ & $107^{\mathrm{ns}}$ & $* 237 \mathrm{bc}^{2}$ & 40 & $* 108$ ef & 59 \\
\hline BR 16 & 45 & 95 & $* 272 \mathrm{abc}$ & 31 & * $139 \mathrm{bcd}$ & 47 \\
\hline CD 201 & 44 & 83 & $* 229 \mathrm{c}$ & 42 & * 131 cde & 50 \\
\hline MSoy 6101 & 48 & 112 & $* 274 \mathrm{abc}$ & 30 & $* 128 \mathrm{de}$ & 51 \\
\hline Bragg & 43 & 106 & $* 281 \mathrm{ab}$ & 28 & * $127 \mathrm{de}$ & 51 \\
\hline BRS 154 & 44 & 120 & * 304 a & 22 & 214 a & 18 \\
\hline Fundacep 38 & 45 & 81 & * 293 a & 25 & $* 168 \mathrm{~b}$ & 35 \\
\hline Fepagro RS 10 & 50 & 104 & $* 240 \mathrm{bc}$ & 39 & $* 088 \mathrm{f}$ & 66 \\
\hline CEPS 9703 & 42 & 110 & $* 288$ a & 26 & $* 158 \mathrm{bc}$ & 39 \\
\hline FT 2000 & 47 & 94 & $* 287 \mathrm{ab}$ & 27 & $* 150 \mathrm{bcd}$ & 42 \\
\hline CEPS 9678 & 45 & 100 & * $276 \mathrm{abc}$ & 30 & * $150 \mathrm{bcd}$ & 42 \\
\hline Erros-padrão & $\pm 1,5$ & $\pm 2,7$ & $\pm 7,1$ & & $\pm 8,2$ & \\
\hline Monocultivo & $45^{\mathrm{ns}}$ & $114^{\mathrm{ns}}$ & 389 & & 258 & \\
\hline
\end{tabular}

${ }^{1 /}$ Redução de MPA aos 60 DAE $\left(\mathrm{R}_{\mathrm{MPA}}\right)$ e de produtividade de grãos $\left(\mathrm{R}_{\mathrm{PROD}}\right)$ em relação ao monocultivo de nabo. ${ }^{\text {ns }}$ Teste $\mathrm{F}$ não-significativo $(\mathrm{p}=0,05)$. Médias seguidas pela mesma letra, comparadas nas colunas, não diferem pelo teste da DMS ( $\mathrm{p}=0,05)$; e, antecedidas por asterisco, diferem do monocultivo de nabo pelo teste de Dunnett $(\mathrm{p}=0,05)$. 
em sistemas de manejo integrado de plantas (Jordan, 1993), dentre os cultivares que detiveram alta habilidade competitiva potencial, CD 201 e RS 10 se destacaram nesse aspecto. $\mathrm{Na}$ presença de nabo, as principais características de planta que diferenciaram esses cultivares dos demais foram maiores estatura de planta, MPA e RMF. Em geral, CD 201 foi mais baixo e acumulou menos matéria seca, porém apresentou RMF superior à de RS 10.

Assim, pode-se concluir que os cultivares de soja respondem diferentemente à interferência do nabo, indicando que existe variabilidade genética que permite selecionar genótipos de soja portadores de habilidade competitiva superior. Os cultivares CD 201 e RS 10 conferem maior poder de supressão ao crescimento de nabo, e menor efeito supressivo verifica-se nos cultivares BRS 154, Fundacep 38 e CEPS 9703. Além disso, a presença de nabo durante os primeiros 60 dias do ciclo da soja reduz estatura de planta, área foliar, matéria seca da parte aérea, emissão e crescimento de ramos e produtividade de grãos de soja.

\section{LITERATURA CITADA}

AARSSEN, L. W. Ecological combining ability and competitive combining ability in plants: toward a general evolutionary theory of coexistence in systems of competition. Am. Natur., v. 122, n. 6, p. 707-731, 1983.

BALBINOT Jr. A. A. et al. Características de plantas de arroz e a habilidade competitiva com plantas daninhas. Planta Daninha, v. 21, v. 2, p. 165-174, 2003.

BEGNA, S. H. et al. Morphology and yield response to weed pressure by corn hybrids differing in canopy architecture. Eur. J. Agron., v. 14, n. 4, p. 293-302, 2001.

BUHLER, D. D. Challenges and opportunities for integrated weed management. Weed Sci., v. 50, n. 3, p. 273-280, 2002.

CATON, B. P.; COPE, A. E.; MORTIMER, M. Growth traits of rice cultivars under severe competition: implications for screening for competitiveness. Field Crops Res., v. 83, n. 1, p. $157-172,2003$.

FLECK, N. G. Competição de azevém (Lolium multiflorum L.) com duas cultivares de trigo. Planta Daninha, v. 3, n. 2, p. 61-67, 1980.
FLECK, N. G. et al. Características de plantas de cultivares de arroz irrigado relacionadas à habilidade competitiva com plantas concorrentes. Planta Daninha, v. 21, n. 1, p. 97-104, 2003.

GIBSON, K. D. et al. Crop traits related to weed suppression in water-seeded rice (Oryza sativa L.). Weed Sci., v. 51, n. 1, p. 87-93, 2003.

GOLDBERG, D. E.; LANDA, K. Competitive effect and response: hierarchies and correlated traits in the early stages of competition. J. Ecol., v. 79, n. 4, p. 1013-1030, 1991.

HAEFELE, S. M. et al. Field screening of diverse rice genotypes for weed competitiveness in irrigated lowland ecosystems. Field Crops Res., v. 88, n. 1, p. 39-56, 2004.

JANNINK, J. L. et al. Index selection for weed suppressive ability in soybean. Crop Sci., v. 40, n. 4, p. 1087-1094, 2000 .

JANNINK, J. L.; JORDAN, N. R.; ORF, J. H. Feasibility of selection for high weed suppressive ability in soybean: absence of tradeoffs between rapid initial growth and sustained later growth. Euphytica, v. 120, n. 2, p. 291-300, 2001.

JORDAN, N. Prospects for weed control through crop interference. Ecol. Applic., v. 3, n. 1, p. 84-91, 1993.

LEMERLE, D. et al. The potential for selecting wheat varieties strongly competitive against weeds. Weed Res., v. 36, n. 6, p. 505-513, 1996.

LINDQUIST, J. L.; MORTENSEN, D. A. Tolerance and velvetleaf (Abutilon theophrasti) suppressive ability of two old and two modern corn (Zea mays) hybrids. Weed Sci., v. 46, n. 5 , p. $569-574,1998$.

LINDQUIST, J. L.; MORTENSEN, D. A. Ecophysiological characteristics of four maize hybrids and Abutilon theophrasti. Weed Res., v. 39, n. 4, p. 271-285, 1999.

McDONALD, G. K. Competitiveness against grass weeds in field pea genotypes. Weed Res., v. 43, n. 1, p. 48-58, 2003.

RADOSEVICH, S. R; HOLT, J.; GHERSA, C. Weed ecology: implications for management. 2.ed. New York: Willey, 1997. $589 \mathrm{p}$.

RAJCAN, I.; SWANTON, C. J. Understanding maize-weed competition: resource competition, light quality and the whole plant. Field Crops Res., v. 71, n. 2, p. 139-150, 2001.

SAS INSTITUTE. User's guide. 4.ed. Cary: 1989. 846 p.

SHILLING, D. G. et al. Effect of soybean (Glycine max) cultivar, tillage and rye (Secale cereale) mulch on sicklepod (Cassia obtusifolia). Weed Technol., v. 9, n. 2, p. 339-342, 1995. 
TRAORÉ, S. et al. Competitive ecophysiology of grain sorghum and Abutilon theophrasti in monoculture and in mixture. Weed Res, v. 42, n. 1, p. 65-75, 2002.

VOLL, E. et al. Competição relativa de espécies de plantas daninhas com dois cultivares de soja. Planta Daninha, v. 20, n. 1, p. 17-24, 2002.
WAX, L. M.; PENDLETON, J. W. Effect of row spacing on weed control in soybeans. Weed Sci., v. 16, n. 4, p. 462-465, 1968.

WORTMANN, C. S. Contribution of bean morphological characteristics to weed suppression. Agron. J., v. 85, n. 4, p. 840-843, 1993. 